\title{
Is there a planetary influence on solar activity?
}

\author{
J. A. Abreu ${ }^{1,2}$, J. Beer ${ }^{2}$, A. Ferriz-Mas ${ }^{3,4}$, K. G. McCracken ${ }^{5}$, and F. Steinhilber ${ }^{2}$ \\ ${ }^{1}$ ETH Zürich Institut für Geophysik, 8092 Zürich, Switzerland \\ e-mail: jose.abreu@erdw.ethz.ch \\ 2 Eawag, Swiss Federal Institute of Aquatic Science and Technology, Postfach 611, 8600 Dübendorf, Switzerland \\ 3 Departamento the Física Aplicada, Universidade de Vigo, Spain \\ ${ }^{4}$ Instituto de Astrofísica de Andalucía (IAA/CSIC), Granada, Spain \\ ${ }^{5}$ University of Maryland, USA
}

Received 12 July 2012 / Accepted 24 September 2012

\begin{abstract}
Context. Understanding the Sun's magnetic activity is important because of its impact on the Earth's environment. Direct observations of the sunspots since 1610 reveal an irregular activity cycle with an average period of about 11 years, which is modulated on longer timescales. Proxies of solar activity such as ${ }^{14} \mathrm{C}$ and ${ }^{10} \mathrm{Be}$ show consistently longer cycles with well-defined periodicities and varying amplitudes. Current models of solar activity assume that the origin and modulation of solar activity lie within the Sun itself; however, correlations between direct solar activity indices and planetary configurations have been reported on many occasions. Since no successful physical mechanism was suggested to explain these correlations, the possible link between planetary motion and solar activity has been largely ignored.

Aims. While energy considerations clearly show that the planets cannot be the direct cause of the solar activity, it remains an open question whether the planets can perturb the operation of the solar dynamo. Here we use a 9400 year solar activity reconstruction derived from cosmogenic radionuclides to test this hypothesis.

Methods. We developed a simple physical model for describing the time-dependent torque exerted by the planets on a non-spherical tachocline and compared the corresponding power spectrum with that of the reconstructed solar activity record.

Results. We find an excellent agreement between the long-term cycles in proxies of solar activity and the periodicities in the planetary torque and also that some periodicities remain phase-locked over 9400 years.

Conclusions. Based on these observations we put forward the idea that the long-term solar magnetic activity is modulated by planetary effects. If correct, our hypothesis has important implications for solar physics and the solar-terrestrial connection.
\end{abstract}

Key words. Sun: dynamo - solar-terrestrial relations - solar wind - Sun: helioseismology - planet-star interactions magnetohydrodynamics (MHD)

\section{Introduction}

The sunspot number provides the longest "direct" record of solar activity, but it is limited to the past 400 years (since the invention of the telescope). Fortunately, solar activity can be reconstructed from cosmogenic radionuclides, thus expanding the history of solar activity for many thousands of years (Solanki et al. 2004; Vonmoos et al. 2006). On entering the Earth's atmosphere, galactic cosmic rays produce cosmogenic radionuclides such as ${ }^{10} \mathrm{Be}$ and ${ }^{14} \mathrm{C}$. The solar magnetic field in the heliosphere modulates the cosmic rays and hence the production rate of these radionuclides. The parameter that best represents the role of the solar magnetic field in deflecting cosmic rays is the solar modulation potential $\phi$, which can be derived from either the ${ }^{10} \mathrm{Be}$ or the ${ }^{14} \mathrm{C}$ production rates. Figure $1 \mathrm{a}$ shows the $\phi$ record (Steinhilber et al. 2012) covering the past $\sim 9400$ yr. This record reveals interesting features: (1) the existence of many Grand Minima; i.e., periods of extended ( $100 \mathrm{y})$ low solar activity similar to the Maunder Minimum; (2) spectral analysis identifies a number of distinct periodicities (Stuiver \& Braziunas 1993), such as $88 \mathrm{yr}$ (Gleissberg), $104 \mathrm{yr}, 150 \mathrm{yr}$, $208 \mathrm{yr}$ (de Vries), $506 \mathrm{yr}, 1000 \mathrm{yr}$ (Eddy), and $2200 \mathrm{yr}$ (Hallstatt) (Figs. 1b and 5a). The stability of the periods of these spectral lines is considerable, but their amplitudes are variable. For instance, the amplitude of the $208 \mathrm{yr}$ cycle is greatest during periods when Grand Minima are more frequent (compare panels $a$ and $b$ in Fig. 1). The periodicity remains strong for up to a millennium: then it almost disappears before reappearing again with the same periodicity (see dashed horizontal line in Fig. 1b). These observations show that the mechanism responsible for generating of the solar magnetic field (called the solar dynamo) operates on a variety of characteristic timescales. The existence of these cycles, which has been known for about 30 years (Sonett 1984) still represents a big challenge for any dynamo model. Current dynamo models, all of which assume that the Sun is an isolated system, are unable to explain these observations. We adopt a different view by regarding the planets and the solar dynamo as two weakly coupled non-linear systems. In this paper we suggest that this coupling is not negligible and show that this hypothesis is able to explain the observed longterm cycles of solar activity.

\section{The planetary hypothesis}

The problem of planetary influence on solar magnetism may be compared to the Milankovitch theory of glaciation, where weak forcings cause strong climate changes. At the time the theory was formulated, there were no experimental data available to test Milankovitch's hypothesis. With the advent of stable isotope measurements from sediment cores, the theory became 


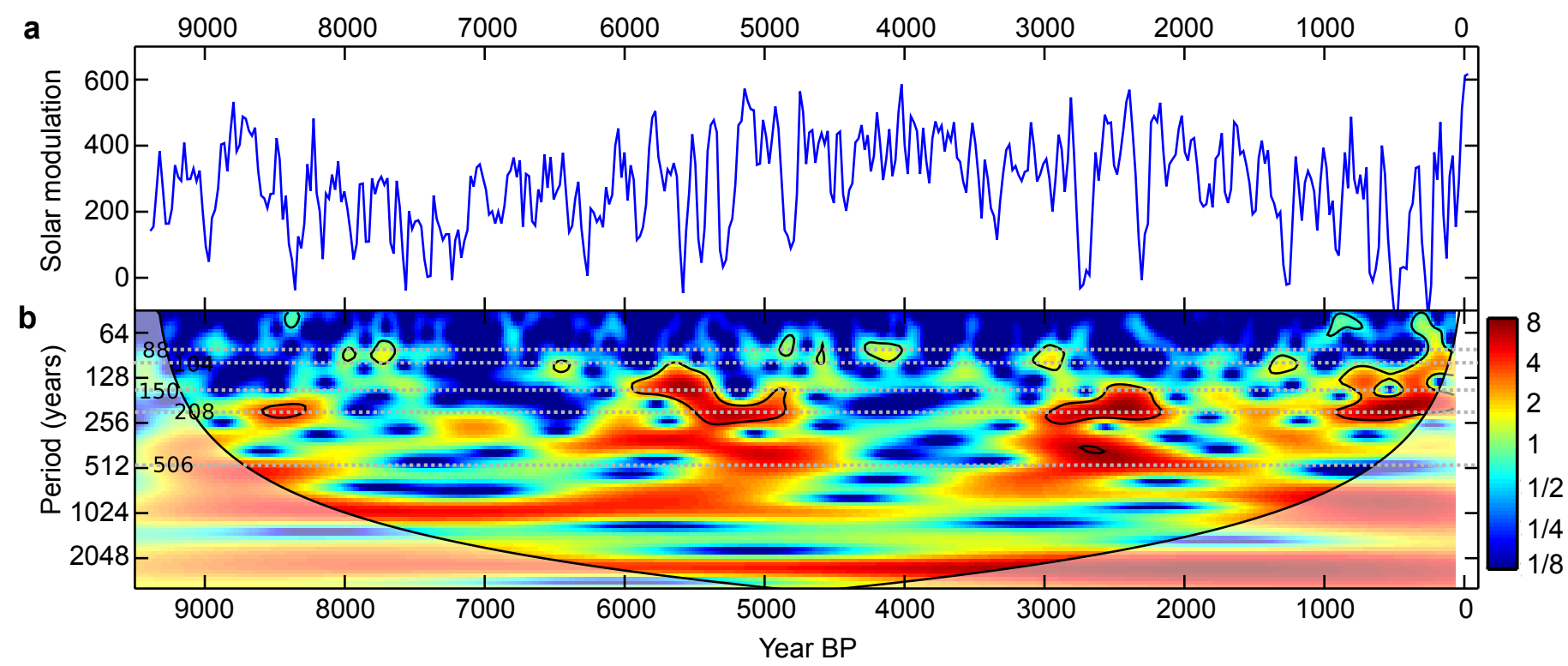

Fig. 1. Properties of solar activity reconstructed from cosmogenic radionuclides. a) Solar activity for the past 9400 years, as specified by the solar modulation potential $\phi$ determined using the cosmogenic radionuclides ${ }^{10} \mathrm{Be}$ and ${ }^{14} \mathrm{C}$ (Steinhilber et al. 2012). b) Corresponding wavelet analysis of solar activity $\phi$ showing the temporal evolution of the amplitudes of the various periodicities. The dashed horizontal lines illustrate the distinct periodicities at $88 \mathrm{yr}, 104 \mathrm{yr}, 150 \mathrm{yr}, 208 \mathrm{yr}$, and $506 \mathrm{yr}$. The red areas indicate high power and blue areas low power. The black contours show the $5 \%$ significance regions (i.e., $95 \%$ confidence level).

widely accepted (Hays et al. 1976). However, it also became clear that without a strong internal amplification mechanism the weak forcing could not cause the well-known sequence of glacial and interglacial periods. Similarly, some internal mechanism in the Sun might also amplify the effect of solar-planetary interaction. The idea that planetary motions may influence the solar activity has a very long history (Kuklin 1976) and seems to have been initiated by the astronomer Rudolf Wolf in the late 1850s (Charbonneau 2002). While energy considerations clearly show that the planets cannot be the direct cause of the solar activity, since this would lead to observable variations in the orbital parameters (Zaqarashvili 1997), it still remains an open question whether the planets can perturb the operation of the solar dynamo. Figure 2a shows a schema of the solar interior. At the interface between the superadiabatic convection zone and the stably stratified radiative interior there is a thin layer of overshooting convection (overshoot layer) in which the temperature gradient is subadiabatic (Fig. 2a, dashed black line). Below the convection zone there is a shear layer known as the tachocline (Spiegel \& Zahn 1992) (Fig. 2b, gray band), which represents a sharp transition between two distinct rotational regimes: the differentially rotating convection zone and the almost rigidly rotating radiative interior. Since the idea was first put forward by Galloway \& Weiss (1981), many solar physicists believe that the tachocline plays a fundamental role in the generation and storage of the toroidal magnetic flux that eventually gives rise to solar active regions (van Ballegooijen 1982; Charbonneau 2010). The relative position between these two boundary layers - one mechanical and one thermal - determines the degree of subadiabaticity of the tachocline and therefore its capability to store magnetic flux tubes (Moreno-Insertis et al. 1992; Ferriz-Mas \& Schüssler 1993, 1995; Fan 2009). The tachocline is also important because tides therein can excite gravity waves (whose restoring force is buoyancy). This is because the buoyancy frequency increases from zero to several cycles per minute as one goes deeper from the convection zone down to the radiative interior; consequently, there is always a

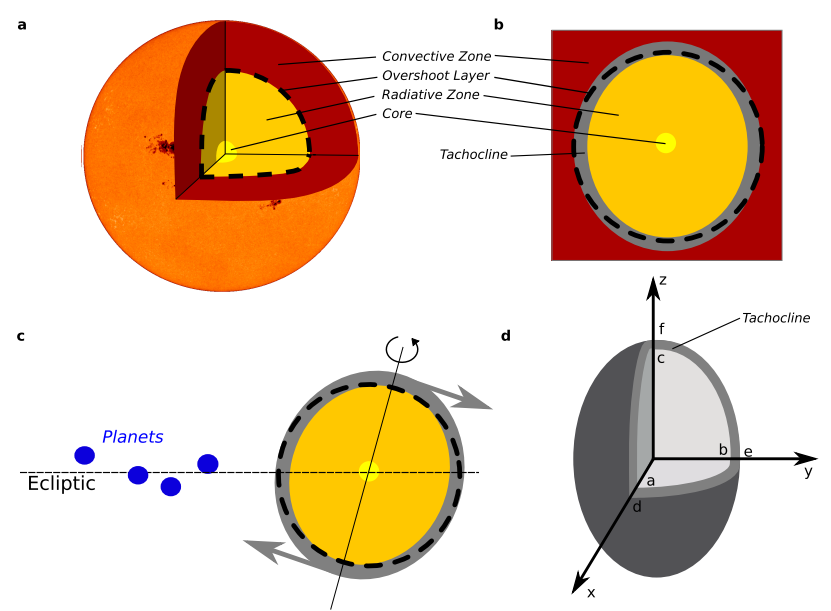

Fig. 2. Internal structure of the Sun, planetary torque, and the solar tachocline. a) Internal structure of the Sun: the core, radiative zone, and convection zone. The overshoot layer (black dashed line) represents the transition between the radiative and the convective zone. b) Two-dimensional representation of the tachocline (gray) modeled as an ellipsoid and the overshoot layer (black dashed line). c) The tachocline (modeled as an ellipsoid), overshoot layer. Note the inclination of the solar rotation axis with respect to the ecliptic. The non-spherical symmetry of the tachocline and the tilt of the solar rotation axis lead to torques. d) Model of the tachocline used for the calculations assuming uniform density between two ellipsoidal shells with semi-major axes $a$, $b, c$ and $d, e, f$. The $z$ direction is parallel to the solar rotation axis.

place between the bottom of the convection zone and the top of the radiative zone where the buoyancy period matches the tidal period (Goldreich \& Nicholson 1989a,b; Goodman \& Dickson 1998; Barker \& Ogilvie 2010). As a result, a net tidal torque is exerted in a small region close to the boundary where the waves are excited (Goldreich \& Nicholson 1989b). Furthermore, the tachocline is inferred to depart significantly from spherical 


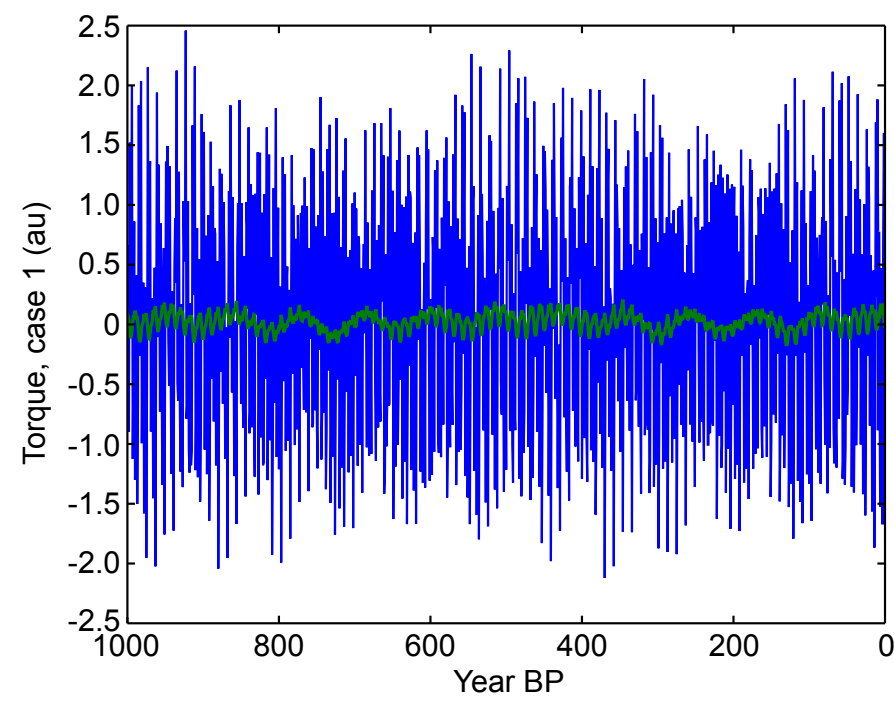

Fig. 3. Planetary torque $|N(t)|$ in abitrary units for case 1 covering the past 1000 years. The blue curve shows the torque averaged over 1 year. The green curve shows the running mean over 22 years.

symmetry: some helioseismological inversions point to a prolate spheroidal geometry (watermelon-shaped) while others favor an oblate (pumpkin-shaped) geometry; for example, Charbonneau et al. (1999) inferred a prolate geometry, with an ellipticity that is a factor of 1000 larger than the solar ellipticity at the photospheric level (Dikpati \& Gilman 2001). Thus a possible coupling mechanism arises from the planetary torques exerted on a nonspherical tachocline (Fig. 2c). If this torque were strong enough to modulate the solar dynamo, it should exhibit features similar to those of solar activity and, in particular, the same periodicities. To test this idea, in the next section we estimate the planetary torque exerted on the tachocline, and compare the results with the observations shown in Fig. 1.

\section{Physical model of planetary influence}

\subsection{Planet-induced torque on the solar tachocline}

To calculate the torque exerted by the planets on the solar tachocline we assume it to be a layer of uniform density between two ellipsoidal shells with semi-major axes $a, b, c$ and $d, e, f$, respectively (see Fig. 2d). The integration domain is restricted to a thin volume between two ellipsoidal shells, which we identify with the tachocline layer. We focus on a thin volume because we expect the excitation of gravity waves only in a small region, as mentioned above.The torque $\boldsymbol{N}_{i}$ exerted by the $i$ th planet with mass $m_{i}$ at position $\boldsymbol{r}_{i}$ from the solar barycenter is given by the following expression (see derivation in Appendix B):

$$
\begin{aligned}
& N_{x, i}=\mathcal{A}_{i} \frac{r_{y, i} \boldsymbol{r}_{z, i}}{\left|\boldsymbol{r}_{i}\right|^{5}}\left[V_{2}\left(e^{2}-f^{2}\right)-V_{1}\left(b^{2}-c^{2}\right)\right], \\
& N_{y, i}=\mathcal{A}_{i} \frac{r_{z, i} r_{x, i}}{\left|\boldsymbol{r}_{i}\right|^{5}}\left[V_{2}\left(f^{2}-d^{2}\right)-V_{1}\left(c^{2}-a^{2}\right)\right], \\
& N_{z, i}=\mathcal{A}_{i} \frac{r_{x, i} r_{y, i}}{\left|\boldsymbol{r}_{i}\right|^{5}}\left[V_{2}\left(d^{2}-e^{2}\right)-V_{1}\left(a^{2}-b^{2}\right)\right],
\end{aligned}
$$

where $\mathcal{A}_{i}=(3 / 5) G \rho m_{i}, G$ being the gravitational constant and $\rho$ the mass density in the tachocline, while $V_{1}=(4 / 3) \pi a b c$ and $V_{2}=(4 / 3) \pi d e f$ are the volumes of the internal and external ellipsoids, respectively. The total torque is the vector sum over all the planets, i.e., $N=\sum_{i} N_{i}$. The torque components are the product of three factors: the first one are the constants $\mathcal{A}_{i}$, the second

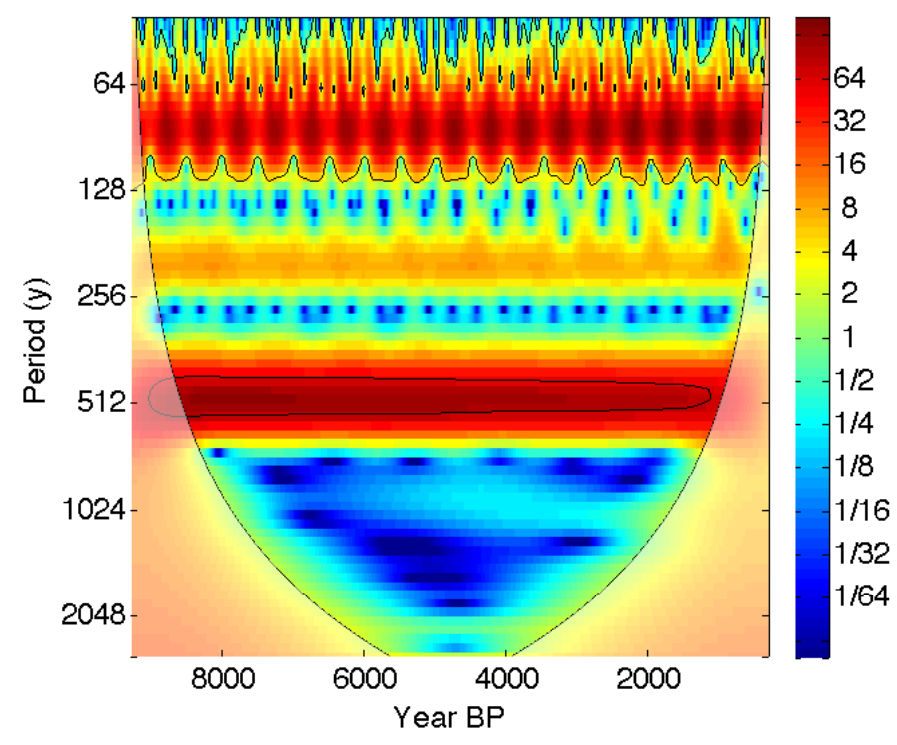

Fig. 4. Wavelet analysis of the planetary torque $|\boldsymbol{N}(t)|$ for case 1 . The periodicities for bands centered at $88 \mathrm{yr}, 208 \mathrm{yr}$ and $506 \mathrm{yr}$ are very stable, but their amplitudes are very small. The red areas indicate high power and blue areas low power.

factor involves the planetary positions and the third one is a geometrical factor (which obviously vanishes for a tachocline contained between two concentric spheres). The geometrical factor may be time-dependent because of changes in the solar magnetic field (Corbard et al. 2001). However, since we do not know how its geometry changes with time, we regard it here as constant. Nevertheless, Eqs. (1)-(3) show that the torque modulus $|N(t)|$ cannot be computed without making additional assumptions regarding the tachocline's geometry. In the present analysis we have explored simple, but still general cases. For simplicity, we assumed spherical geometry for the inner boundary, i.e. $a=b=c$. We analyzed the following cases. (1) $e=f$ (i.e., the component $N_{x}$ vanishes). (2) $f=d$ (i.e, the component $N_{y}$ vanishes; this case is equivalent to (1) by symmetry), and (3) $d=e$ (i.e., $N_{z}$ vanishes). For each case, we computed the corresponding torque modulus $|N(t)|$ (the planetary torque corresponding to case 1 as a function of time is shown in Fig. 3, while Fig. 4 shows the corresponding wavelet analysis). Figure 5 displays the Fourier spectra of solar activity $(\phi)$, and the torque for the three cases. Visual inspection of the spectra shows a striking similarity. The well-known Gleissberg ( $88 \mathrm{yr}$ ) and de Vries (208 yr) cycles are clearly seen in both, together with additional periodicities around $104 \mathrm{yr}, 150 \mathrm{yr}$, and $506 \mathrm{yr}$. Indeed, all these spectral lines are also observed in different records of cosmogenic radionuclides. Table 1 shows these five common spectral lines observed in ${ }^{10} \mathrm{Be}$ from the GRIP ice core (Vonmoos et al. 2006), ${ }^{14} \mathrm{C}$ from tree rings (Reimer et al. 2009) and $\phi$ (see also Appendix A). Monte Carlo tests with one million $\phi$ records consisting of random numbers show that the probability of finding the same five spectral lines by chance is about $10^{-7}$ to $10^{-11}$, respectively (see next section). We note that the spectrum is independent of the specific geometry adopted. To identify regions in the time-frequency space where the two time series co-vary, we carried out a wavelet coherence (WTC) analysis (Grinsted et al. 2004) of $\phi$ and of the torque. The WTC corresponding to case (1) is shown in Fig. 6, whereas the results for cases (2) and (3) are shown in Figs. 7 and 8, respectively. Case (1) and case (2) reveal the same results. Interestingly, the results for case (3) do not agree with the observations as well as cases (1) and (2). 


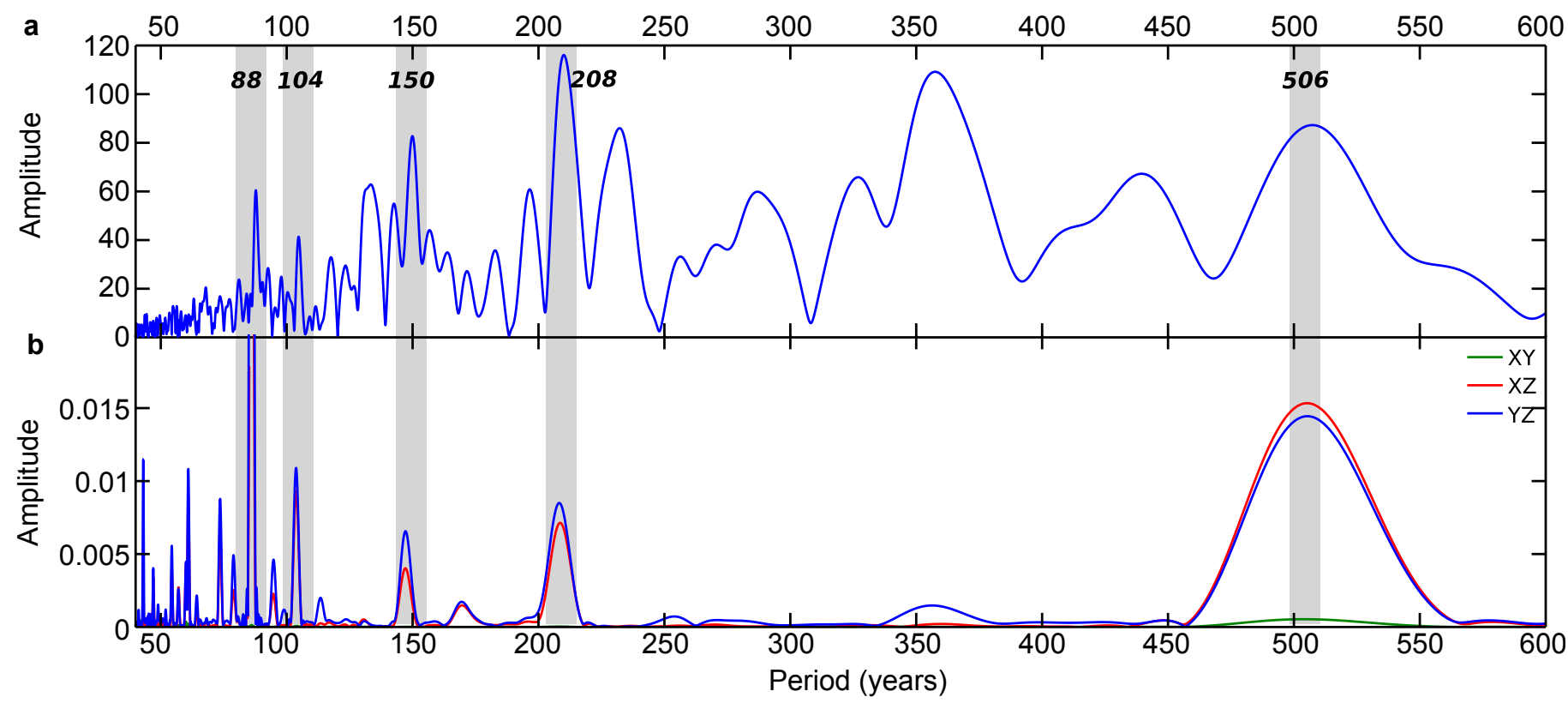

Fig. 5. Comparison between solar activity and planetary torque in the frequency domain. a) Fourier spectrum of the solar activity quantified by the solar modulation potential $\phi$. b) Fourier spectrum of the annually averaged torque modulus $|N(t)|$ (blue corresponds to case 1 , red to case 2 , and green to case 3). The spectra display significant peaks with very similar periodicities: the $88 \mathrm{yr}$ Gleissberg and the $208 \mathrm{yr}$ de Vries cycles are the most prominent, but periodicities around $104 \mathrm{yr}, 150 \mathrm{yr}$, and $506 \mathrm{yr}$ are also seen.

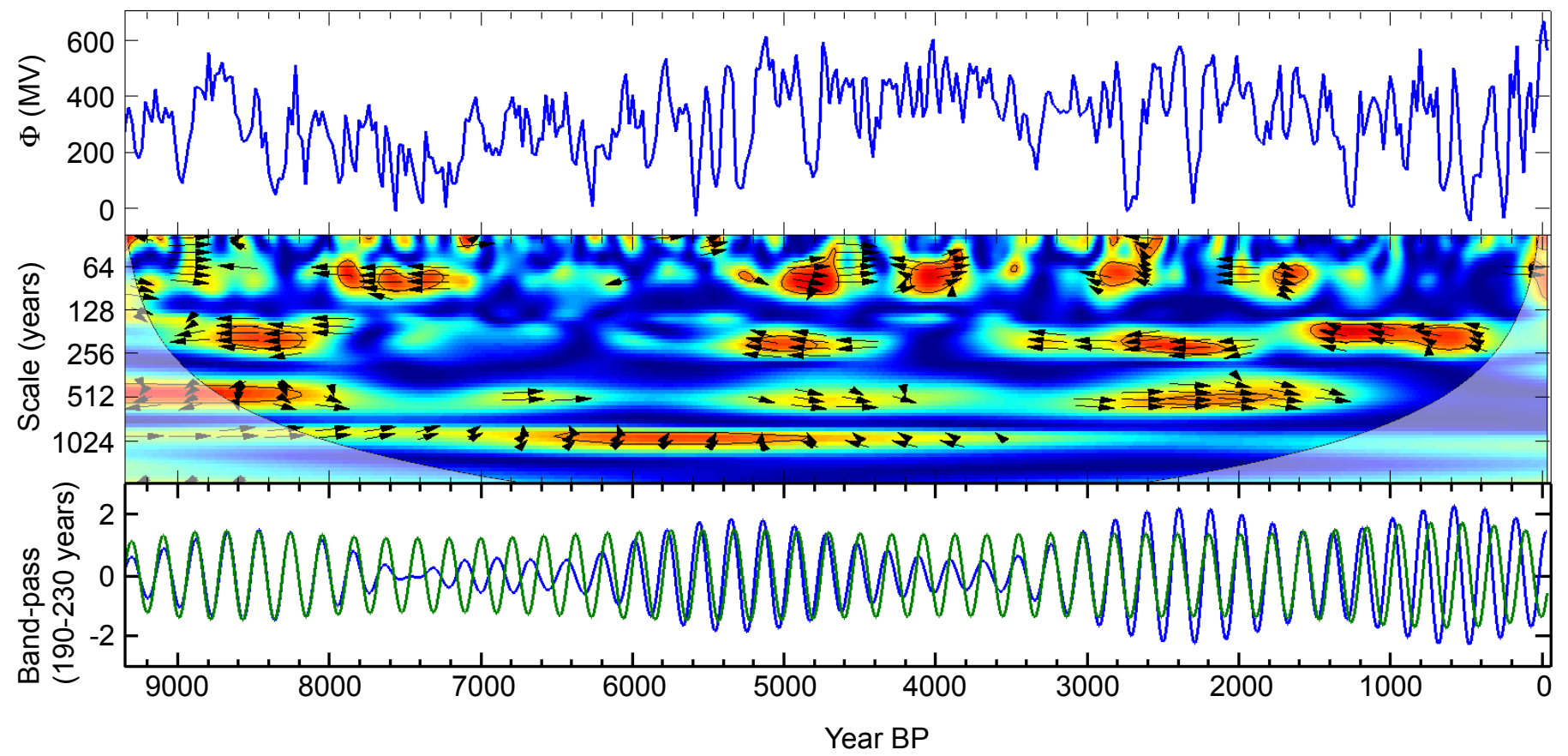

Fig. 6. Comparison between solar activity and planetary torque corresponding to case 1 in both frequency and time domain. a) Solar activity $\phi$ reconstructed from cosmogenic radionuclides (Steinhilber et al. 2012). b) Wavelet coherence between observation $(\phi)$ and torque $(|N(t)|)$. Arrows indicate the relative phase between both series (Grinsted et al. 2004) (pointing to left, anti-phase; to right in phase). The constant phase between $\phi$ and the torque for the bands centered at $208 \mathrm{yr}$ and $506 \mathrm{yr}$ are an indication of phase locking. The black contours show the 5\% significance regions (95\% confidence level). c) Band-pass-filtered annually averaged torque (green curve) along with $\phi$ (blue curve - inverted scale-) around the 208 yr periodicity (de Vries). Note that the intervals where forcing (torque) and the response $(\phi)$ are in anti-phase correspond to time periods when Grand Minima are more frequent, which is also evident in the wavelet analysis, supporting the assumption of a physical coupling between the planets and the Sun.

Figure $6 \mathrm{~b}$ shows the WTC of $\phi$ and the torque for case (1). Arrows indicate the relative phase between the two time series. We observe high coherence distributed in several period bands. In the following we focus on the $208 \mathrm{yr}$ cycle. The strong coherence for the $208 \mathrm{yr}$ band with a constant relative phase shows that solar activity and the planetary torque are phase locked. Note that phase locking arises naturally when parametric autoresonance occurs (Khain \& Meerson 2001). We also observe intermittence in the signal. Time intervals of high coherence correspond to periods when the amplitude of the $208 \mathrm{yr}$ cycle is 


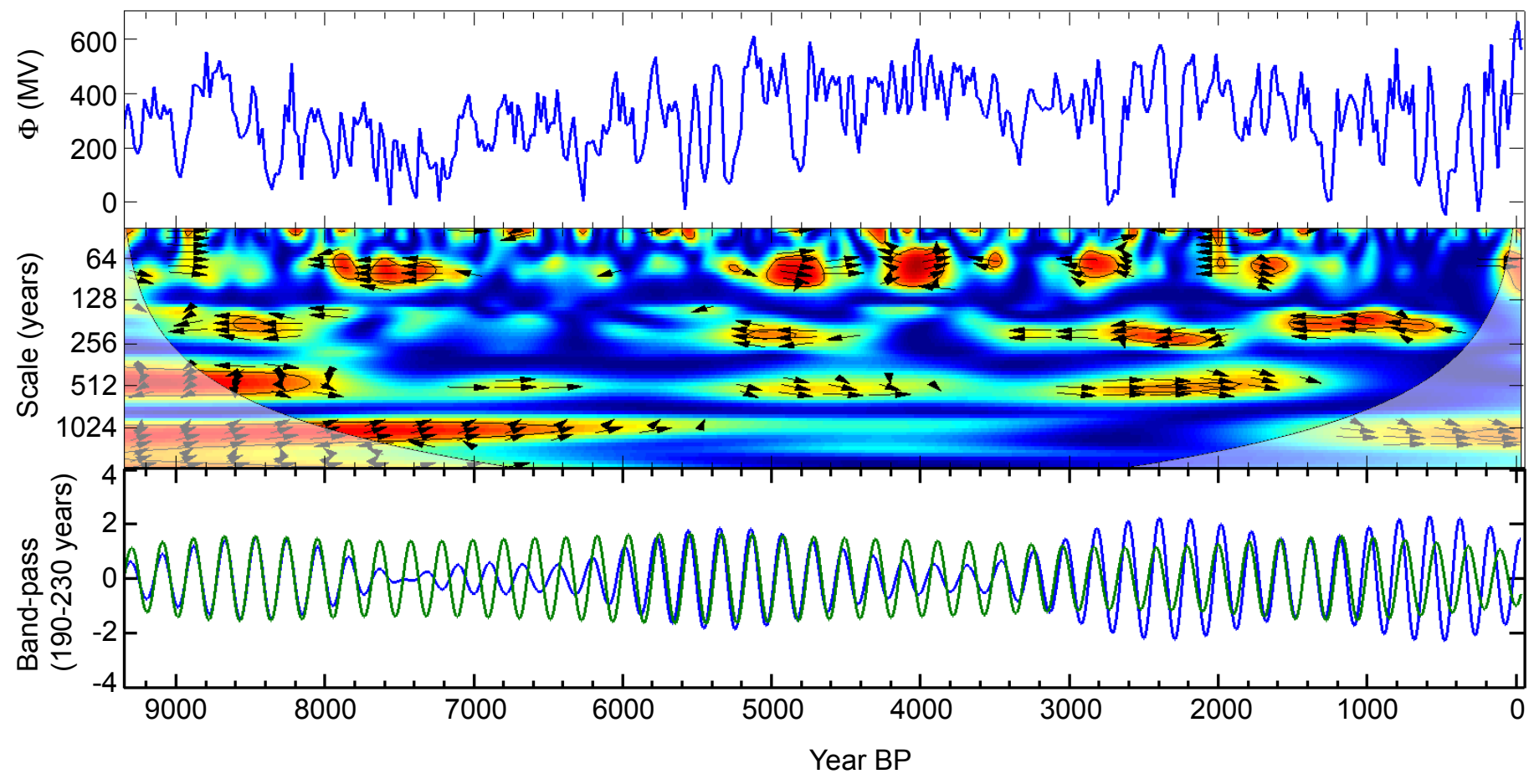

Fig. 7. Comparison between solar activity and planetary torque corresponding to case 2 in both frequency and time domain. a) Solar modulation potential $\phi$ reconstructed from cosmogenic radionuclides (Steinhilber et al. 2012). b) Wavelet coherence (WTC) between $\phi$ and the torque $|N(t)|$. c) Band-pass filtered annually averaged torque (green) along with $\phi$ (blue-inverted scale) around the 208 yr de Vries cycle. Results for case (2) agree with case (1) as well as with the observations. The red areas indicate high coherence and blue areas low coherence. The black contours show the $5 \%$ significance regions (95\% confidence level).

large, followed by periods of no coherence when the amplitude of the $208 \mathrm{yr}$ cycle is weak. Figure $6 \mathrm{c}$ shows the result of applying a band-pass filter to $\phi$ (blue line) and to the torque (green line) around the de Vries cycle (208 yr). The relative timing between the two time series is quite good overall. The two $208 \mathrm{yr}$ bandpass filtered time series are in anti-phase when the amplitude of the de Vries cycle is large and Grand Minima are more frequent. Similar results showing significant coherence are obtained for other bands, although with less stable relative phases. The analysis for case (3) leads to a much weaker coherence and a less stable phase around $208 \mathrm{yr}$ (Fig. 8). The corresponding band pass filtering confirms the poor agreement between $\phi$ and case 3 . The difference between cases $(1,2)$ and $(3)$ points to a surprising result: a non-vanishing $N_{z}$ component provides the best agreement between torque and solar activity. This would imply the existence of a torque component parallel to the solar spin; in other words, the geometry of the tachocline would be a triaxial ellipsoid (either oblate or prolate). This result is consistent with the current knowledge of the structure of the tachocline as derived from global helioseismological inversion. At the present time, global helioseismology neither provides information about the longitudinal structure of the tachocline, nor can it distinguish between the hemispheres (ChristensenDalsgaard \& Thompson 2007; Thompson 2010). However, the geometry of the tachocline is complex and likely to be time dependent (Antia \& Basu 2011). Certainly, the structural asymmetry inferred herein is consistent with the existence of active longitudes (Berdyugina \& Usoskin 2003).

\subsection{Significance of the spectral agreement}

In this section we investigate the important question whether the good agreement between $\phi$ and the torque could occur by chance (see Appendix A for a more detailed discussion). To estimate the probability for such a coincidence we used a random generator and produced artificial time series of the same length $(7000 \mathrm{yr})$. Then we calculated the Fourier spectrum, selected the periods between $40 \mathrm{yr}$ and $600 \mathrm{yr}$ and tested whether one or several of the 20 strongest lines fall within one or several windows defined for the five selected spectral lines in Fig. 5. The results of the analysis of a million random time series with a white spectrum are summarized in Table 2. The second column shows how many of the simulated time series had lines falling into the defined windows. The fourth column lists the number of coincidences with the previous lines $(1-2,1-3,1-4,1-5)$. Although none of our simulations yielded all five lines simultaneously, the probability of such a case can be calculated from the third column, which is $\sim 5 \times 10^{-7}$. Repeating the test using time series with a red instead of a white spectrum leads to a probability of $10^{-11}$ (see Table 3). In other words, there is highly statistically significant evidence for a causal relationship between the power spectra of the planetary torque on the Sun and the observed magnetic activity at the solar surface as derived from cosmogenic radionuclides. As suggested by the anonymous referee, using alternate solar systems (i.e., a different number of planets with different masses, eccentricities, orbital inclinations, etc.) would be an even more stringent test, but this would require computing very many synthetic ephemerides, which is beyond the scope of the present work.

\section{Discussion and conclusions}

The excellent spectral agreement between the planetary tidal effects acting on the tachocline and the solar magnetic activity is surprising, because until now the tidal coupling has been considered to be negligible. In Appendix A we show that the possibility of an accidental coincidence can be ruled out. We therefore 


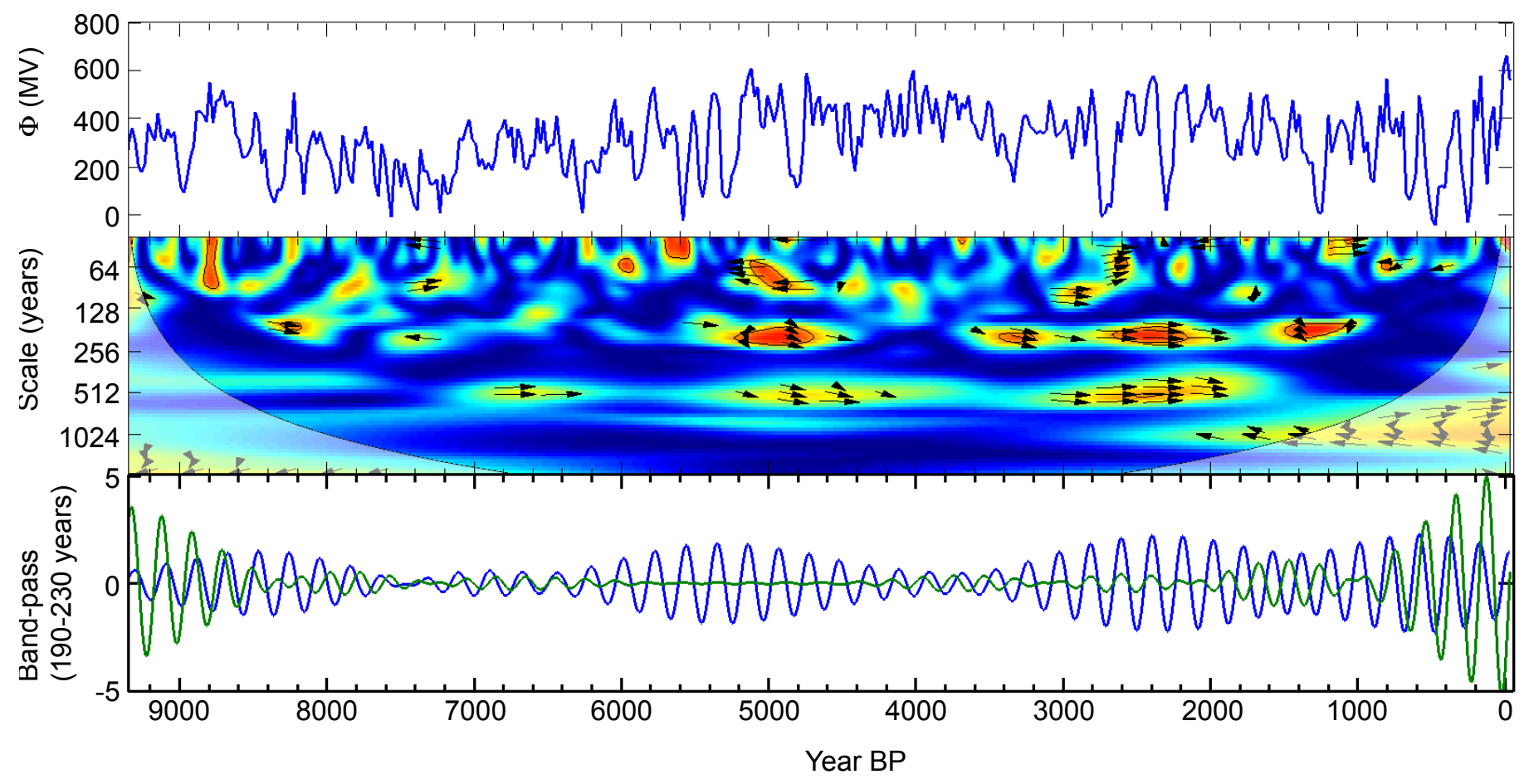

Fig. 8. Comparison between solar activity and planetary torque corresponding to case 3 in both frequency and time domain. a) Solar modulation potential $\phi$ reconstructed from cosmogenic radionuclides (Steinhilber et al. 2012). b) Wavelet coherence (WTC) between $\phi$ and the torque $|\boldsymbol{N}(t)|$. c) Band-pass filtered annually averaged torque (green) along with $\phi$ (blue-inverted scale) around the 208 yr (de Vries) cycle. Compared to the cases (1) and (2) the results of case (3) agree less well with the observations. The analysis leads to a much smaller coherence and a less stable phase around $208 \mathrm{yr}$. The band pass filtering confirms the poor agreement between the torque and $\phi$ for this case. The red areas indicate high coherence and blue areas low coherence. The black contours show the 5\% significance regions (95\% confidence level).

Table 1. Coincidence of spectral lines in several records.

\begin{tabular}{lcccc}
\hline \hline Period window & ${ }^{10}$ Be concentr. & ${ }^{14}$ C production & $\phi$ & Torque \\
\hline $85-89$ & 86 & 88 & 88 & 86 \\
$103-106$ & 104 & 105 & 105 & 104 \\
$146-151$ & 148 & 150 & 150 & 147 \\
$206-210$ & 207 & 209 & 209 & 209 \\
$503-515$ & 504 & 512 & 508 & 504 \\
\hline
\end{tabular}

Notes. The first column shows the chosen narrow windows for the five spectral lines observed in Fig. 5. Within the chosen windows all records of ${ }^{10} \mathrm{Be}$ from the GRIP ice core (Vonmoos et al. 2006), ${ }^{14} \mathrm{C}$ from tree rings (Reimer et al. 2009) and the $\phi$ record (Steinhilber et al. 2012) show the same spectral lines.

Table 2. Results of the Monte Carlo runs simulating random time series of $\phi$ with a white spectrum.

\begin{tabular}{lcccc}
\hline \hline Period window & Counts & Probability & Coincidence & Coincidence probability \\
\hline $85-89$ & 143095 & 0.143 & & Observed/calculated \\
$103-106$ & 103835 & 0.104 & 13697 & $0.0137 / 0.0149$ \\
$146-151$ & 168087 & 0.168 & 1886 & $0.0019 / 0.0025$ \\
$206-210$ & 189785 & 0.189 & 261 & $2.61 \times 10^{-4} / 4.74 \times 10^{-4}$ \\
$503-515$ & 1064 & 0.0011 & 0 & $0 / 5.04 \times 10^{-7}$ \\
\hline
\end{tabular}

Table 3. Results of the Monte Carlo runs simulating random time series of $\phi$ with a red spectrum.

\begin{tabular}{lcccc}
\hline \hline Period window & Counts & Probability & Coincidence & Coincidence probability \\
\hline $503-513$ & 91440 & 0.091 & & Observed/calculated \\
$206-210$ & 546884 & 0.547 & 48533 & $0.0485 / 0.0500$ \\
$146-151$ & 453977 & 0.454 & 20011 & $0.0200 / 0.0227$ \\
$103-106$ & 1016 & 0.0010 & 14 & $1.4 \times 10^{-5} / 2.31 \times 10^{-5}$ \\
$85-89$ & 2 & $2.0 \times 10^{-6}$ & 0 & $0 / 4.61 \times 10^{-11}$ \\
\hline
\end{tabular}


suggest that a planetary modulation of the solar activity does take place on multidecadal to centennial time scales. Since the tidal acceleration of the planets on the solar interior is small, it is not clear how the tidal effects could influence the internal dynamics of the Sun. We here advance an argument of plausibility on how this mechanism might happen. The crucial point in our argument is that the tachocline plays a key role in the dynamo process (Galloway \& Weiss 1981; van Ballegooijen 1982; Charbonneau 2010). The tachocline is on the one hand a layer of strong shear, which is a basic ingredient of the dynamo process. On the other hand, it more or less coincides spatially with the a layer of overshooting convection at the bottom of the convection zone. The overshoot layer is thought to be crucial for the storage and amplification of the magnetic flux tubes that eventually erupt at the solar photosphere to form active regions. One of the key factors determining the storage capacity of the overshoot layer is the superadiabaticity $\delta$, which is a dimensionless measure of the stratification of the specific entropy in a medium. If we call $\nabla$ the logarithmic temperature gradient (using pressure as the radial coordinate) and $\nabla_{\text {ad }}$ the corresponding value of $\nabla$ for a homoentropic medium (same specific entropy everywhere), then $\delta=\nabla-\nabla_{\text {ad }}$. In a radiative zone $\delta<0$ (stable stratification), in a convection zone $\delta>0$ (unstable stratification) and somewhere at the bottom of the convection zone $\delta$ changes sign. $\delta$ is negative and very small in the overshoot layer, where it is believed that strong toroidal flux tubes $\left(\sim 10^{5} \mathrm{G}\right)$ are stored prior to the eruption of the sunspots. The maximum field strength of a flux tube that can be stored at a given latitude in the overshoot layer depends very sensitively on the value of $\delta$, which is about $-10^{-4}$ to $-10^{-5}$. Small variations in $\delta$ of about $-10^{-4}$ may decide whether a flux tube becomes unstable at $2 \times 10^{4} \mathrm{G}$ or at $10^{5} \mathrm{G}$. This makes a great difference, because flux tubes that do not reach a strength close to $10^{5} \mathrm{G}$ before entering the convection zone cannot reach the solar surface as a coherent structure and therefore cannot form sunspots (Moreno-Insertis et al. 1995). Thus the tiny tidal effect could influence the magnetic storage capacity of the tachocline by modifying the stratification of entropy $(\delta)$, and thereby altering the maximum field strength of the flux tubes that can be stored there. The exact way in which this tiny modification ( 1 part in $10^{4}$ or $10^{5}$ ) of the entropy stratification is produced by the tidal forces is the missing link in the theory, but we can think of a resonance effect mediated by gravity waves. Since this coupling takes place in the tachocline, the tidally excited gravity waves (Goldreich \& Nicholson 1989a,b; Goodman \& Dickson 1998; Barker \& Ogilvie 2010) may be modified by the shear of the environment. Shear may be an important ingredient because, under appropriate conditions, it allows a disturbance to grow by systematic extraction of energy from the flow (Craik 1968). A detailed investigation of these potential mechanisms is beyond the scope of this paper and needs to be addressed in the future. We emphasize the potential of monitoring solar-like stars with exoplanets, which could provide an independent test of the hypothesis (Perryman \& Schulze-Hartung 2011). Additional motivation comes from the observation of tidally interacting binary stellar systems, which show enhanced magnetic activity compared to isolated stars (Schrijver \& Zwaan 1991; Welsh et al. 2011).

If correct, our planetary hypothesis may have important implications for solar physics and the solar-terrestrial connection. The Sun and its planets were formed 4.5 billion years ago from a giant molecular cloud. The present form of the solar system is the result of a long-term evolution controlled by interactions such as gravitation and collisions. Here we suggest that a full understanding of the long-term solar magnetic activity can only be achieved by considering the influence of the planets on the Sun and allowing for internal amplification mechanisms. As a first step in this process we have proposed a simple model describing planetary torques acting on a non-spherical solar tachocline.

Acknowledgements. We acknowledge financial support by National Centre of Competence in Research climate, Swiss climate research, and by the Swiss National Science Foundation under Grant CRSI122-130642 (Future and Past Solar Influence on the Terrestrial Climate). The US National Science Grant 1050002 supported the University of Maryland component of this work. We thank Markus Roth (Kiepenheuer-Institut für Sonnnenphysik, Freiburg) for his advice in connection with the solar tachocline.The work of AFM has been partially funded by the Spanish Ministerio de Ciencia e Innovación and Ministerio de Economía y Competitividad, through Projects No. AYA2009-14105-C06 and AYA2011-29833-C06, including European FEDER funds. We would like to thank the anonymous referee for valuable and constructive suggestions. Wavelet software was provided by C. Torrence and G. Compo, and is available at URL: http: //atoc. colorado.edu/research/wavelets/.

\section{Appendix A: Simulation of spectral lines}

There are many well-defined spectral lines in solar activity which can be seen in time series derived from ${ }^{14} \mathrm{C}$ in tree rings and ${ }^{10} \mathrm{Be}$ in polar ice cores. Some of the most pronounced ones are the Gleissberg cycle $(\sim 88 \mathrm{yr})$, the de Vries or Suess cycle ( $\sim 208 \mathrm{yr}$ ), and the unnamed cycles with periodicities of $\sim 104 \mathrm{yr}$ and $\sim 150$ yr. These cycles were identified in the first ${ }^{14} \mathrm{C}$ data (Damon \& Jirikowic 1992; Sonett 1984; Stuiver \& Braziunas 1989). With the continuous improvement of precision in radiocarbon measurements and the extension of the tree ring record up to 12400 years these spectral lines are now very well established. They are complemented by ${ }^{10} \mathrm{Be}$ measurements in polar ice cores from Greenland as well as from Antarctica, and are well-defined in the new $9400 \mathrm{yr}$ long reconstruction of solar variability (Steinhilber et al. 2012). ${ }^{14} \mathrm{C}$ and ${ }^{10} \mathrm{Be}$ are both produced by cosmic rays in the atmosphere, but have completely different geochemical properties $\left({ }^{14} \mathrm{C}\right.$ enters the carbon cycle by forming $\mathrm{CO}_{2}$, while ${ }^{10} \mathrm{Be}$ becomes attached to aerosols and is removed from the atmosphere mainly by wet deposition). In the following we show that the Fourier spectra of all records show consistent results for the past period 300-9400 BP (we exclude the past $300 \mathrm{yr}$, which are disturbed by fossil fuel burning and the nuclear bomb tests; $9400 \mathrm{yr}$ is determined by the length of the ${ }^{10} \mathrm{Be}$ record). Then we compare these spectra with the spectrum of the torque exerted by the planets on the tachocline for the same period. We observe that five of the strongest spectral lines in all three records agree very well. Finally, we determine the probability that these five lines agree by chance using a Monte Carlo technique. We conclude that the chance of a random coincidence is about $5 \times 10^{-7}$.

\section{A.1. Short description of the method}

We used four time series covering the same time period (300-9400 BP). All records are interpolated to one year using a cubic spline $\left({ }^{10} \mathrm{Be},{ }^{14} \mathrm{C}, \phi\right)$ or averaged over one year (torque):

1. ${ }^{10} \mathrm{Be}$ from the GRIP ice core (Vonmoos et al. 2006);

2. ${ }^{14} \mathrm{C}$ production rate derived from the ${ }^{14} \mathrm{C}$ INTCAL09 record (Reimer et al. 2009) using a carbon cycle model (Muscheler et al. 2007). The spectra of the measured $\Delta^{14} \mathrm{C}$ and the calculated ${ }^{14} \mathrm{C}$ production rate are very similar for the periodicities, but the amplitudes are different because of the dampening effect of the carbon system;

3. $\phi$ record based on ${ }^{10} \mathrm{Be}$ from the GRIP core (Greenland), ${ }^{10} \mathrm{Be}$ from the EDML ice core (Antarctica) and the ${ }^{14} \mathrm{C}$ production record (Steinhilber et al. 2012); 


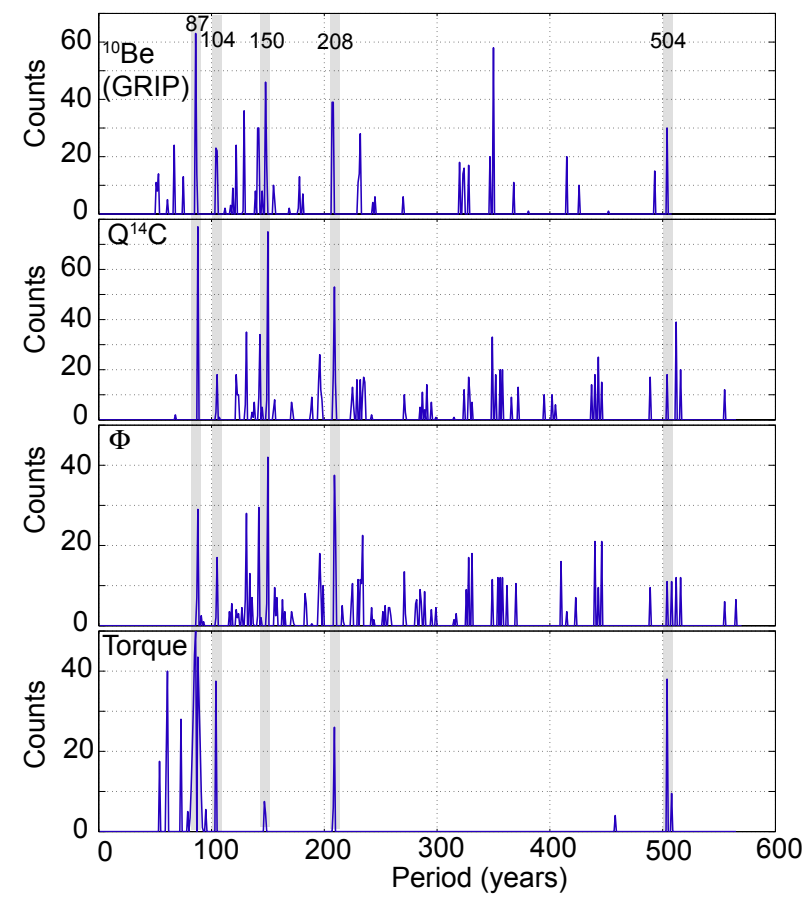

Fig. A.1. Spectra of four different records for the period 300-9400 years BP. a) ${ }^{10} \mathrm{Be}$ from the GRIP ice core in Greenland (Vonmoos et al. 2006). b) ${ }^{14} \mathrm{C}$ production rate derived from the ${ }^{14} \mathrm{C}$ INTCAL09 record (Reimer et al. 2009). c) $\phi$ record based on ${ }^{10} \mathrm{Be}$ records from GRIP (Greenland) and Drowning Maud Land (Antarctica) and the ${ }^{14} \mathrm{C}$ production rate (Steinhilber et al. 2012). d) Calculated torque based on planetary positions.

4. Torque exerted by the planets using the NASA Jet Propulsion Laboratory Ephemeris DE-408 J2000 (see Appendix C) and Eq. (1) of our model.

All four records were treated alike. A 7000 year time window was moved in steps of $500 \mathrm{yr}$ through the time series. For each step a fast Fourier transform was calculated and a list of the periodicities between $40 \mathrm{yr}$ and $600 \mathrm{yr}$ was generated. From this list, the 20 strongest lines were selected. Each line was rounded to the full year and weighted according to its ranking (first line by a factor 20 , weakest line by a factor 1 ). The sum of the spectra for these four time windows is shown for the four records in Fig. A.1. Table 1 shows the five common spectral lines that were selected. For each line we defined a window given in the first column that we consider representative for this line. Note the remarkable similarity of the periodicities. The strongest discrepancy occurs in the last line (504-512 yr). Inspection shows that there is also a line at $504 \mathrm{yr}$ in the $\phi$ record.

The next point we address is whether the good agreement between ${ }^{10} \mathrm{Be},{ }^{14} \mathrm{C}$ and $\phi$ with the torque could occur by chance. To estimate the probability for such a coincidence we used a random generator and produced one million artificial time series of the same length $(7000 \mathrm{yr})$ with a white and a red spectrum. Then we calculated the Fourier spectrum, selected the periods between $40 \mathrm{yr}$ and $600 \mathrm{yr}$ and tested whether one or several of the 20 strongest lines fall within one or several windows defined for the five selected spectral lines in Table 1 . The results of the analysis of a million random time series with a white spectrum are summarized in Table 2. The second column shows how many of the simulated time series had lines falling into the defined windows. The fourth column lists the number of coincidences with the previous lines $(1-2,1-3,1-4,1-5)$. Although none of our

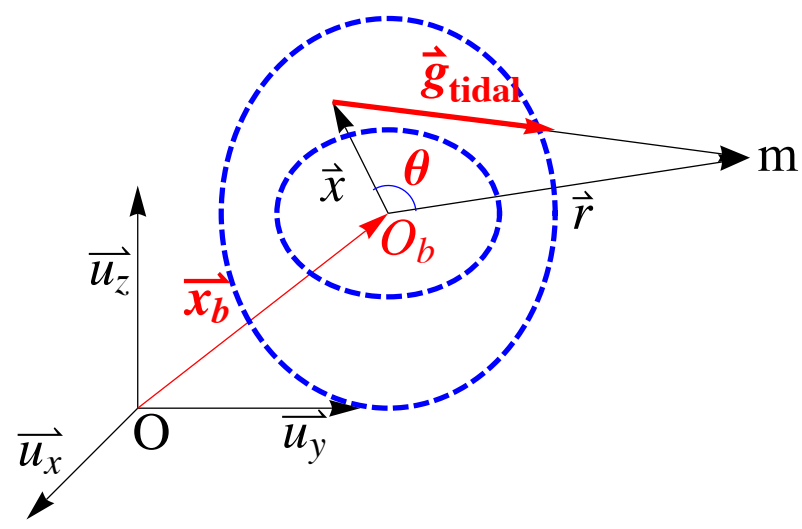

Fig. B.1. Schematic illustration of the tidal acceleration exerted by a planet of mass $m$ on a point $\boldsymbol{x}$ in the solar interior. $O$ represents the center of mass of the solar system. $O_{b}$ represents the solar center of mass. $\boldsymbol{x}_{b}$ is the instantaneous position of the solar center of mass with respect to the center of mass of the solar system. The vector $\boldsymbol{r}$ represents the instantaneous distance between the planet and the solar center of mass. $\left\{\boldsymbol{u}_{i}\right\}$ is the basis of the equator J2000 reference frame (explained in text). The dashed blue lines represent the boundaries of the solar tachocline, which are assumed to be ellipsoids.

simulations yielded all five lines simultaneously, the probability of such a case can be calculated from the third column. The calculated probability that all five lines occur simultaneously is $\sim 5 \times 10^{-7}$, which is consistent with this never occurring during the one million test runs. It is interesting to note that the counts for the last line (503-515 yr) critically depend on the length of the time series. Using a shorter time series of 6000 or fewer years does no longer provide any counts, which reduces the probability significantly to $<10^{-11}$, while the radionuclide spectra still contain the same spectral lines. For a red spectrum the probability is $\sim 5 \times 10^{-11}$ (see Table 3 ).

\section{Appendix B: Derivation of Eq. (1)}

Here we derive the expression for the tidal torque exerted by the planets on a fluid shell of uniform density bounded by two concentric ellipsoids. Hereafter we will refer to this region as $R$. By "tidal acceleration" we mean the differential acceleration caused by a planet of mass $m$ acting upon an arbitrary point of the Sun at a distance $\boldsymbol{x}$ with respect to the solar center of mass. We assume the planets to be mass points. From Newton's law of gravitation:

$\boldsymbol{g}(\boldsymbol{x}, \boldsymbol{r})=G m \frac{\boldsymbol{r}-\boldsymbol{x}}{|\boldsymbol{r}-\boldsymbol{x}|^{3}}$

where $\boldsymbol{r}$ is the distance between the solar center of mass and the planet. Thus the acceleration exerted by a planet at the solar center of mass is obtained by letting $\boldsymbol{x}=\mathbf{0}$. The tidal acceleration exerted by a planet of mass $m$ at an arbitrary point $\boldsymbol{x}$ is defined as (see Fig. B.1)

$\boldsymbol{g}_{\text {tidal }}(\boldsymbol{x}, \boldsymbol{r}) \equiv \boldsymbol{g}(\boldsymbol{x}, \boldsymbol{r})-\boldsymbol{g}(\mathbf{0}, \boldsymbol{r})$.

Thus the torque per unit of volume exerted by a planet of mass $m$ on a point $\boldsymbol{x}$ with respect to the solar center of mass is

$\boldsymbol{n}(\boldsymbol{x}, \boldsymbol{r})=\rho \boldsymbol{x} \wedge \boldsymbol{g}_{\mathrm{tidal}}(\boldsymbol{x}, \boldsymbol{r})=\rho G m(\boldsymbol{x} \wedge \boldsymbol{r})\left(\frac{1}{|\boldsymbol{r}-\boldsymbol{x}|^{3}}-\frac{1}{|\boldsymbol{r}|^{3}}\right)$, 
where $\rho(\boldsymbol{x})$ is the solar mass density at $\boldsymbol{x}$. The term $|\boldsymbol{r}-\boldsymbol{x}|^{-3}$ can be written as

$|\boldsymbol{r}-\boldsymbol{x}|^{-3}=|\boldsymbol{r}|^{-3}\left\{1+\epsilon^{2}-2 \epsilon \cos \theta\right\}^{-3 / 2}$,

where $\epsilon \equiv|\boldsymbol{x}| /|\boldsymbol{r}|$ and $\theta$ is the angle between the $\boldsymbol{x}$ and $\boldsymbol{r}$. We can use the fact that $\epsilon \ll 1$ and expand Eq. (B.4) (up to second order) as

$|\boldsymbol{r}-\boldsymbol{x}|^{-3} \simeq|\boldsymbol{r}|^{-3}\{1+3 \epsilon \cos \theta\}$.

Finally the torque reads

$\boldsymbol{n}(\boldsymbol{x}, \boldsymbol{r})=3 \rho G m(\boldsymbol{x} \wedge \boldsymbol{r}) \frac{\boldsymbol{x} \cdot \boldsymbol{r}}{|\boldsymbol{r}|^{5}}$.

Next we assume the tachocline to be a fluid shell of uniform density bounded by two concentric ellipsoids (see Fig. B.1). We estimate the tidal torque by integrating the vector field given by Eq. (B.6) in the region $R$ :

$N=\int_{R} \boldsymbol{n}(\boldsymbol{x}, \boldsymbol{r}) \mathrm{d}^{3} \boldsymbol{x}$

with $R=\left\{(x, y, z) \mid 1 \leq \frac{x^{2}}{a^{2}}+\frac{y^{2}}{b^{2}}+\frac{z^{2}}{c^{2}}\right.$ and $\left.1 \geq \frac{x^{2}}{d^{2}}+\frac{y^{2}}{e^{2}}+\frac{z^{2}}{f^{2}}\right\}$.

Assuming constant density and assuming that the positions of the planets are independent of $\boldsymbol{x}$, we can compute the torque,

$N_{x, i}=\mathcal{A}_{i} \frac{r_{y, i} r_{z, i}}{\left|\boldsymbol{r}_{i}\right|^{5}}\left[V_{2}\left(e^{2}-f^{2}\right)-V_{1}\left(b^{2}-c^{2}\right)\right]$,

$N_{y, i}=\mathcal{A}_{i} \frac{r_{z, i} r_{x, i}}{\left|\boldsymbol{r}_{i}\right|^{5}}\left[V_{2}\left(f^{2}-d^{2}\right)-V_{1}\left(c^{2}-a^{2}\right)\right]$,

$N_{z, i}=\mathcal{A}_{i} \frac{r_{x, i} r_{y, i}}{\left|\boldsymbol{r}_{i}\right|^{5}}\left[V_{2}\left(d^{2}-e^{2}\right)-V_{1}\left(a^{2}-b^{2}\right)\right]$,

where $\mathcal{A}_{i}=(3 / 5) G \rho m_{i}, V_{1}=(4 / 3) \pi a b c$ and $V_{2}=(4 / 3) \pi d e f$ are the volumes of the internal and external ellipsoids, respectively. The total torque is the vector sum over all the planets, i.e., $N=\sum_{i} N_{i}$.

\section{Appendix C: Reference frame}

The coordinates of the planets were obtained from NASA Jet Propulsion Laboratory Ephemerides DE-408 J2000. We used the equator $\mathbf{J} 2000$ reference frame. This reference frame uses the Earth equator at epoch J2000 (i.e., 1. January 2000 $11: 58: 55,816$ UTC) as the fundamental plane. The $x$-axis points along the $\mathbf{J} 2000$ vernal equinox, the $z$-axis is the mean north pole, and the $y$-axis completes the right-hand orthonormal basis. As origin we chose the solar center of mass $\left(O_{b}\right.$ in Fig. C. 1$)$. The point $O_{b}$ together with the right-hand orthonormal basis $\left\{\boldsymbol{u}_{i}\right\}$ specify the Cartersian coordinate frame depicted in Fig. C.1. For convenience, we defined a new coordinate frame $\left(O_{b},\left\{\boldsymbol{e}_{i}\right\}\right)$. This system uses as z-axis the solar rotation axis $\boldsymbol{\Omega}$. The basis $\left\{\boldsymbol{e}_{i}\right\}$ is constructed as follows:

$\boldsymbol{e}_{z} \equiv \frac{\boldsymbol{\Omega}}{\|\boldsymbol{\Omega}\|}, \quad \boldsymbol{e}_{x} \equiv \frac{\boldsymbol{e}_{z} \wedge \boldsymbol{u}_{z}}{\left\|\boldsymbol{e}_{z} \wedge \boldsymbol{u}_{z}\right\|}=\frac{\boldsymbol{e}_{z} \wedge \boldsymbol{u}_{z}}{\sin (\beta)}, \quad \boldsymbol{e}_{y} \equiv \boldsymbol{e}_{z} \wedge \boldsymbol{e}_{x}$,

where $\beta$ is the angle between $\boldsymbol{u}_{z}$ and $\boldsymbol{\Omega}$. The coordinates of the solar spin $\boldsymbol{\Omega}$ in $\mathrm{J} 2000$ are (Fig. C. 1 ) $\delta=63.87^{\circ}$ and $\alpha=286.13^{\circ}$ (Fränz \& Harper 2002). Note that we assumed $\boldsymbol{\Omega}$ to be constant in time.

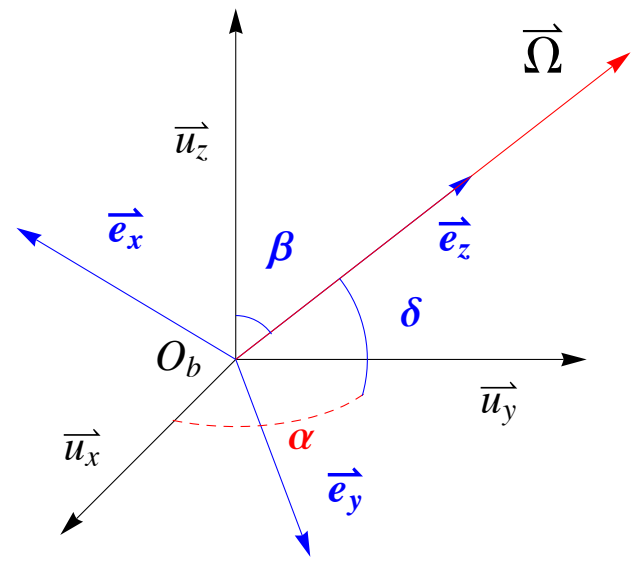

Fig. C.1. Reference frame employed. $\left\{\boldsymbol{u}_{i}\right\}$ is the basis of the equator $\mathrm{J} 2000$ reference frame (explained in the text). $\mathrm{O}_{b}$ represents the position of the solar center of mass, which we chose as origin. For convenience, we introduced a new coordinate frame $\left(O_{b},\left\{\boldsymbol{e}_{i}\right\}\right)$. This system uses as $z$-axis the solar rotation axis $\boldsymbol{\Omega}$. Here $\delta$ and $\alpha$ are the declination and right ascension of the solar rotation axis at epoch $\mathbf{J} 2000$.

\section{References}

Antia, H. M., \& Basu, S. 2011, ApJ, 735, L45

Barker, A. J., \& Ogilvie, G. I. 2010, MNRAS, 404, 1849

Berdyugina, S. V., \& Usoskin, I. G. 2003, A\&A, 405, 1121

Charbonneau, P. 2002, J. Hist. Astron., 33, 351

Charbonneau, P. 2010, Liv. Rev. Sol. Phys., 7, 3

Charbonneau, P., Christensen-Dalsgaard, J., Henning, R., et al. 1999, ApJ, 527, 445

Christensen-Dalsgaard, J., \& Thompson, M. J. 2007, in The Solar Tachocline, eds. D. W. Hughes, R. Rosner, \& N. O. Weiss, 53

Corbard, T., Jiménez-Reyes, S. J., Tomczyk, S., Dikpati, M., \& Gilman, P. 2001, in SOHO 10/GONG 2000 Workshop: Helio- and Asteroseismology at the Dawn of the Millennium, eds. A. Wilson, \& P. L. Pallé, ESA SP, 464, 265

Craik, A. D. D. 1968, J. Fluid Mech., 34, 531

Damon, P. E., \& Jirikowic, J. L. 1992, Radiocarbon, 34, 199

Dikpati, M., \& Gilman, P. A. 2001, ApJ, 552, 348

Fan, Y. 2009, Liv. Rev. Sol. Phys., 6, 4

Ferriz-Mas, A., \& Schüssler, M. 1993, Geophys. Astrophys. Fluid Dyn., 72, 209

Ferriz-Mas, A., \& Schüssler, M. 1995, Geophys. Astrophys. Fluid Dyn., 81, 233

Fränz, M., \& Harper, D. 2002, Planet. Space Sci., 50, 217

Galloway, D. J., \& Weiss, N. O. 1981, ApJ, 243, 945

Goldreich, P., \& Nicholson, P. D. 1989a, ApJ, 342, 1079

Goldreich, P., \& Nicholson, P. D. 1989b, ApJ, 342, 1075

Goodman, J., \& Dickson, E. S. 1998, ApJ, 507, 938

Grinsted, A., Moore, J. C., \& Jevrejeva, S. 2004, Nonlinear Processes Geophys., 11,561

Hays, J. D., Imbrie, J., \& Shackleton, N. J. 1976, Science, 194, 1121

Khain, E., \& Meerson, B. 2001, Phys. Rev. E, 64, 036619

Kuklin, G. V. 1976, in Basic Mechanisms of Solar Activity, eds. V. Bumba, \& J. Kleczek, IAU Symp., 71, 147

Moreno-Insertis, F., Schuessler, M., \& Ferriz-Mas, A. 1992, A\&A, 264, 686

Moreno-Insertis, F., Caligari, P., \& Schuessler, M. 1995, ApJ, 452, 894

Muscheler, R., Joos, F., Beer, J., et al. 2007, Quaternary Sci. Rev., 26, 82

Perryman, M. A. C., \& Schulze-Hartung, T. 2011, A\&A, 525, A65

Reimer, P. J., Baillie, M. G. L., Bard, E., et al. 2009, Radiocarbon, 51, 1111

Schrijver, C. J., \& Zwaan, C. 1991, A\&A, 251, 183

Solanki, S. K., Usoskin, I. G., Kromer, B., Schussler, M., \& Beer, J. 2004, Nature, 431, 1084

Sonett, C. P. 1984, Rev. Geophys., 22, 239

Spiegel, E. A., \& Zahn, J. 1992, A\&A, 265, 106

Steinhilber, F., Abreu, J. A., Beer, J., et al. 2012, PNAS, 109, 5967

Stuiver, M., \& Braziunas, T. F. 1989, Nature, 338, 405

Stuiver, M., \& Braziunas, T. F. 1993, The Holocene, 3, 289

Thompson, M. J. 2010, in SOHO-23: Understanding a Peculiar Solar Minimum, eds. S. R. Cranmer, J. T. Hoeksema, \& J. L. Kohl, ASP, 428, 23

van Ballegooijen, A. A. 1982, A\&A, 113, 99

Vonmoos, M., Beer, J., \& Muscheler, R. 2006, J. Geophys. Res., 111, A10105

Welsh, W. F., Orosz, J. A., Aerts, C., et al. 2011, ApJS, 197, 4

Zaqarashvili, T. V. 1997, ApJ, 487, 930 